\title{
Traditional Chinese patent medicine for bile reflux gastritis: a systematic review and network meta-analysis
}

\author{
Meijun $\mathrm{He}^{1}$, Qun Wang ${ }^{1}$, Lin Liu ${ }^{2}$, Guang Bai ${ }^{2}$ \\ ${ }^{1}$ Liaoning University of Traditional Chinese Medicine, Shenyang, China; ${ }^{2}$ Department of Gastroenterology, Affiliated Hospital of Liaoning \\ University of Traditional Chinese Medicine, Shenyang, China \\ Contributions: (I) Conception and design: G Bai; (II) Administrative support: G Bai; (III) Provision of study materials or patients: M He, Q Wang; (IV) \\ Collection and assembly of data: M He; (V) Data analysis and interpretation: L Liu, M He; (VI) Manuscript writing: All authors; (VII) Final approval \\ of manuscript: All authors. \\ Correspondence to: Guang Bai. Department of Gastroenterology, Affiliated Hospital of Liaoning University of Traditional Chinese Medicine, No. 33, \\ Beiling Street, Huanggu District, Shenyang 110033, China. Email: Baiguang3767@outlook.com.
}

Background: Traditional Chinese Patent Medicine (TCPM) is widely used in the treatment of bile reflux gastritis (BRG). However, there is still a lack of research evaluating the efficacy of specific drugs. Thus, we conducted a reticulated meta-analysis to compare the efficacy of TCPMs in the treatment of BRG.

Methods: We searched the China National Knowledge Infrastructure (CNKI), PubMed, Web of Science, and the Wanfang, and Embase databases, as of February 2021, for publications on the treatment of BRG with Chinese patent medicines in randomized controlled trials (RCTs). The main outcome indicator was the effective rate. The secondary outcome indicators were recurrence rate, traditional Chinese medicine (TCM) symptom score, and gastroscopic mucosal score. The Cochrane bias risk assessment tool was used to evaluate the research quality, and RevMan software (5.2) and Stata software (15.0) were used for the network metaanalysis.

Results: A total of 24 studies were included in the meta-analysis. In total, 2,417 patients were included in the meta-analysis, comprising 1,222 patients in the treatment group and 1,195 patients in the control group. The results of the network meta-analysis showed that Weiyankang capsules combined with hydrotalcite had the best effect in the treatment of bile reflux among the 14 interventions. Among the 5 studies that reported recurrence rates, patients administered Shugan Hewei pills had the lowest recurrence rate. A direct comparison showed that TCPMs or TCPMs combined with Western medicines had certain advantages in improving the scores of traditional Chinese medicine symptoms and mucosal scores under gastroscopy.

Discussion: Among all the Chinese patent medicines examined, Weiyankang capsules combined with hydrotalcite appeared to be the best choice for the treatment of BRG. However, due to limitations related to the quantity and quality of the research, more high-quality research needs to be conducted in the future to gather additional evidence.

Trial Registration: The protocol of this network meta-analysis was registered in PROSPERO with ID CRD42021247873.

Keywords: Traditional Chinese patent medicine; bile reflux gastritis (BRG); network meta-analysis; randomized controlled trials (RCTs)

Submitted Apr 30, 2021. Accepted for publication Jun 29, 2021.

doi: 10.21037/apm-21-1307

View this article at: https://dx.doi.org/10.21037/apm-21-1307 


\section{Introduction}

Bile reflux gastritis (BRG) refers to inflammatory lesions of the gastric mucosa caused by the reflux of bile into the stomach (1). Clinically, stomach pain, acid reflux, bitter mouth, nausea, and vomiting are the main symptoms observed following the development of BRG. The most common gastroscopic changes are congestion, edema, erosion, intestinal metaplasia, and gastric polyps (2). BRG is a type of chronic gastritis, accounting for about $22.6 \%$ of chronic gastritis (3). Retrospective analysis showed that the detection rate of BRG in the Chinese population is $11.3 \%$ and that the rate is significantly higher among women (4). The etiology and pathogenesis of BRG are complex and involve many mechanisms, such as gastrointestinal motility and neuroendocrine and pathological changes (5). The condition reoccurs easily, often lingers, and is difficult to fully heal. Long-term bile reflux can cause diseases, such as esophageal adenocarcinoma and gastric cancer, which seriously endanger human health. At present, the most common treatments in Western medicine are gastric mucosal protective agents, prokinetic drugs, and proton pump inhibitors (6). The effects of each treatment are different; however, the treatments themselves are often prolonged and lead to unhealed lesions, and relapse can easily occur when treatment is stopped.

Many types of TCPMs are used in the treatment of BRG in clinical practice, and studies have shown that these medicines have promising results $(7,8)$. Lou et al. treated BRG patients with Weikang capsules and found that the total effective rate in the experimental group was significantly higher than in the control group treated with Cisapride tablets $(\mathrm{P}<0.05)(9)$. Xie et al. treated 60 BRG patients with Danweishu granules, achieving a total effective rate of $91.7 \%$, which was significantly higher than the $65 \%$ total effective rate in the control group patients treated with Domperidone tablets, Ranitidine capsules and Sucralfate tablets $(\mathrm{P}<0.05)(10)$. Zhuang et al. treated BRG patients in an experimental and the control group with Danweining granules and Domperidone tablets combined with Hydrotalcite, respectively. The total effective treatment rate in the experimental group was $93.33 \%$, which was significantly higher and in the control group of $66.67 \%(\mathrm{P}<0.01)(11)$.

However, to date, little research has been conducted comparing these different medicines to determine which formula is the best at treating the disease. This study used a network meta-analysis to compare the most commonly used TCPMs in the treatment of BRG in clinical practice. The use of the clinical curative effect in this study provides an evidence base for clinical use.

We present the following article in accordance with the PRISMA reporting checklist (available at https://dx.doi. org/10.21037/apm-21-1307).

\section{Methods}

\section{Search strategy}

The publications used in the meta-analysis were identified by searches of the China National Knowledge Infrastructure (CNKI), PubMed, Web of Science, and the Wanfang, VIP, and Embase databases. The search period ranged from the beginning of the construction of the databases to February 2021, and the search language was Chinese or English. The following terms were used in the Medical Subject Headings (MeSH) of each database: "bile reflux gastritis" AND "Weiyankang capsule" OR "Weisu granule" OR "Qizhi Weitong granule" OR "Xiaoyan Lidan dropping pill" OR "Shugan pill" OR "Danweining granule" OR "Weikang capsule."

\section{Inclusion and exclusion criteria}

To be eligible for inclusion in the meta-analysis, the studies had to meet the following inclusion criteria: (I) be a randomized controlled trial (RCT); (II) have study participants that comprised patients with a clear diagnosis of BRG (there were no limitations in relation to race, age, or sex); (III) have an observation group treated with a Chinese patent medicine or a Chinese patent medicine combined with a Western medicine, and a control group treated with a Western medicine or a Western medicine combined with a Chinese patent medicine (in that particular order). Notably, the Chinese patent medicine had to have a clear preparation manufacturer and approval number; and (IV) have a primary outcome indicator of clinical effectiveness, and secondary outcome indicators of the TCM symptom score, incidence of adverse reactions, and recurrence rate. Conversely, studies were excluded from the meta-analysis, if they met any of the following inclusion criteria: (I) used Chinese medicine combined with acupuncture and other therapies; and/or (II) did not report efficiency.

\section{Data extraction}

NoteExpress software was used to classify the documents 


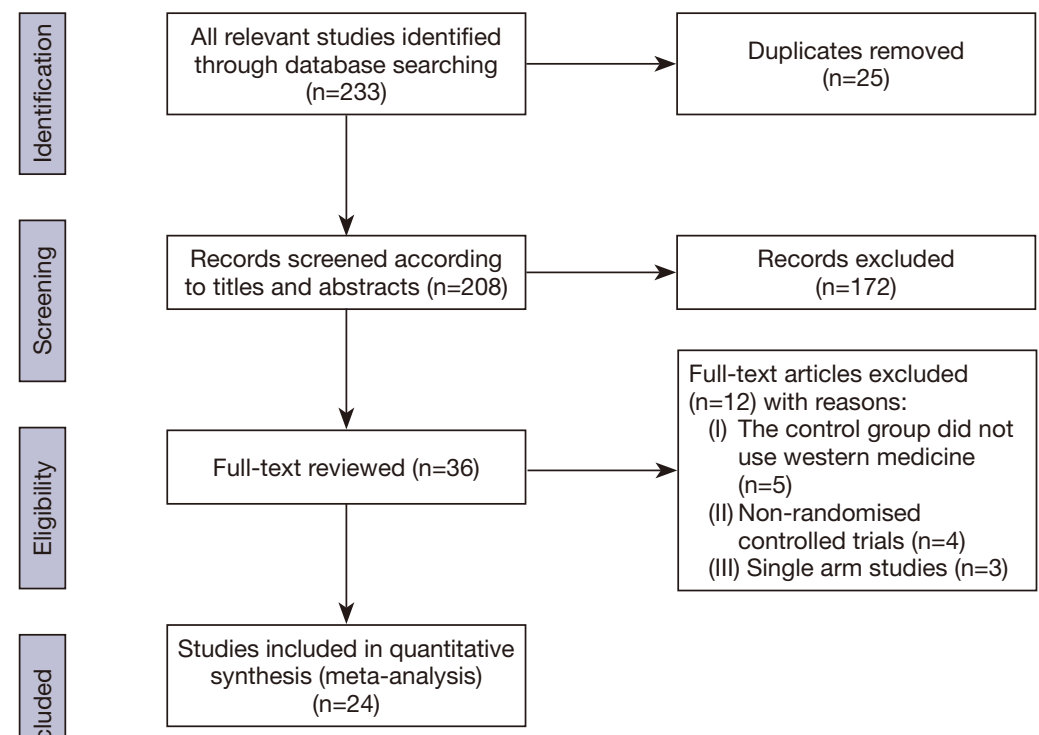

Figure 1 Study selection.

retrieved in the searches of the databases. First, duplicate publications were removed, and the publications were screened according to the established inclusion and exclusion criteria. For consistency purposes, the same two researchers independently screened the publications and extracted the data. If the researchers' opinions differed, a third professional evaluator made the judgment. The main contents of the extracted data included the title, author, year of publication, age, sex ratio, sample size, disease course, and intervention measures.

\section{Risk of bias assessment}

Two reviewers independently used the Cochrane risk of bias tool to evaluate the included studies. A total of 7 items were included, and each item related to the risk of bias evaluation was identified as either high risk, low risk, or unclear. Disagreements were resolved by a third researcher.

\section{Statistical analysis}

RevMan software (5.2) was used to draw the risk bias maps and conduct the meta-analysis. Stata software (15.0) was used to perform a mesh meta-analysis under the consistency model. Binary variables were expressed using odds ratios (ORs) and 95\% confidence intervals (CIs). Continuous variables are represented as mean difference
(MD) and 95\% CI. I ${ }^{2}$ was used to assess heterogeneity, in which $\mathrm{I}^{2}$ values greater than $50 \%$ indicated substantial statistical heterogeneity (12). Stata 15.0 was used to sort the curative effect and draw the cumulative probability sorting chart to obtain the surface under the cumulative ranking (SUCRA). Indirect comparisons of different interventions were completed by drawing an evidence network diagram. Finally, Stata 15.0, was used to draw a "comparisoncorrection" funnel chart to identify whether there was a small sample effect.

\section{Results}

\section{Included studies}

Based on the search criteria, 223 articles were included in the preliminary screening, and 25 duplicate articles were removed. After reading the titles and abstracts, 172 documents were excluded. The remaining 36 articles were read in full, and 24 RCTs that met the requirements were included [see the PRISMA flow diagram (13) in Figure 1].

\section{Characteristics and quality assessment}

A total of 24 qualified, double-arm RCTs were included $(9,11,14-35)$. We assessed the quality of the included studies 


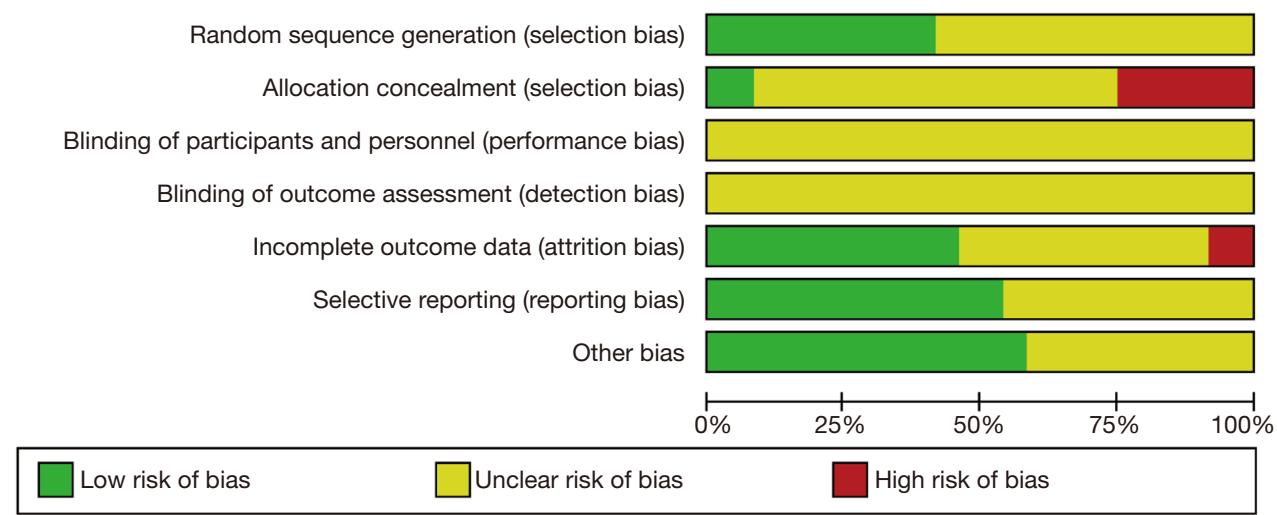

Figure 2 Quality assessment of the risk of bias for each included study.

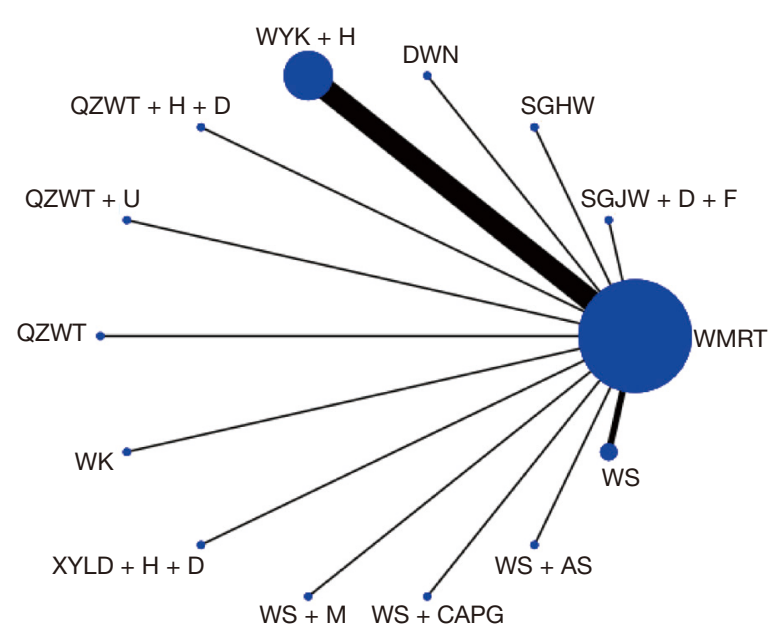

Figure 3 Network of eligible comparisons of the efficacy of treatments.

using the Cochrane risk of bias tool. Each evaluation principle was divided into "high risk," "low risk" or "unclear" (see Figure 2). A total of 14 interventions were included in this study (see Figure 3). The total sample size was 2,417 cases, comprising 1,222 cases in the experimental group and 1,195 cases in the control group. The following 8 Chinese patent medicines were included: Shugan Jianwei pills, Shugan Hewei pills, Danweining granules, Weiyankang capsules, Qizhi Weitong granules, Weikang capsules, Xiaoyan Lidan dropping pills, and Weisu granules. The publication year period for the publications ranged from 1998 to 2021. All patients were diagnosed with BRG according to clear diagnostic criteria, and the studies were conducted in China. The basic characteristics of the included studies are listed in Table 1.

\section{Network meta-analysis}

\section{Primary outcomes}

All of the studies reported efficient primary outcome indicators in relation to 15 interventions (see the network plot in Figure 3). The comparison between the intervention measures of all the studies and conventional Western medicine treatments is shown in Figure 4. The results showed that intervention measures of Chinese patent medicines or Chinese patent medicines combined with Western medicines had significantly better efficacy than conventional Western medicine treatments $[\mathrm{OR}=4.26,95 \%$ CI (3.28, 5.53)].

The network results are compared in Figure 5. There was no closed loop between the interventions; that is, there was no direct comparison between the interventions. All pairwise comparisons between the interventions came from indirect comparisons; thus, the statistical analysis could be performed directly under the consistency model. A total of 105 pairwise comparisons with total effective rates were produced, of which only 1 comparison had a statistically significant difference. Treatment with Weiyankang capsules combined with hydrotalcite had a better curative effect than conventional Western medicines [OR $=1.80,95 \%$ CI $(1.30,2.29)]$. The order of the effective SUCRA values of the TCPMs for the treatment of BRG was as follows: WYK + H(Weiyankang capsules + Hydrotalcite) (SUCRA =73.2), DWN(Danweining granules) (SUCRA =72.8), SGJW + D + F (Shugan Jianwei pills + Domperidone + Famotidine) $($ SUCRA $=69.9), \mathrm{WS}+\mathrm{M}($ Weisu granules + 


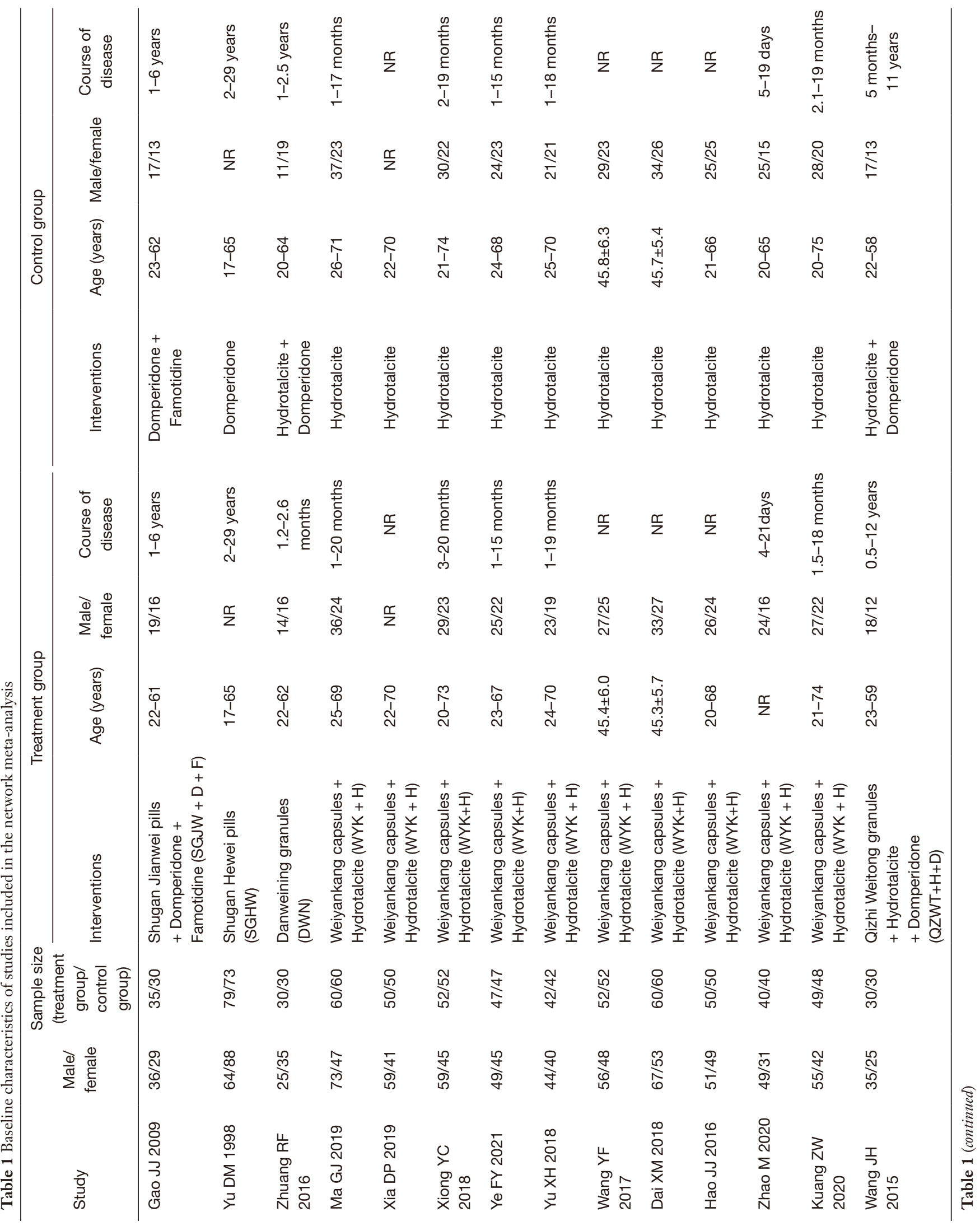




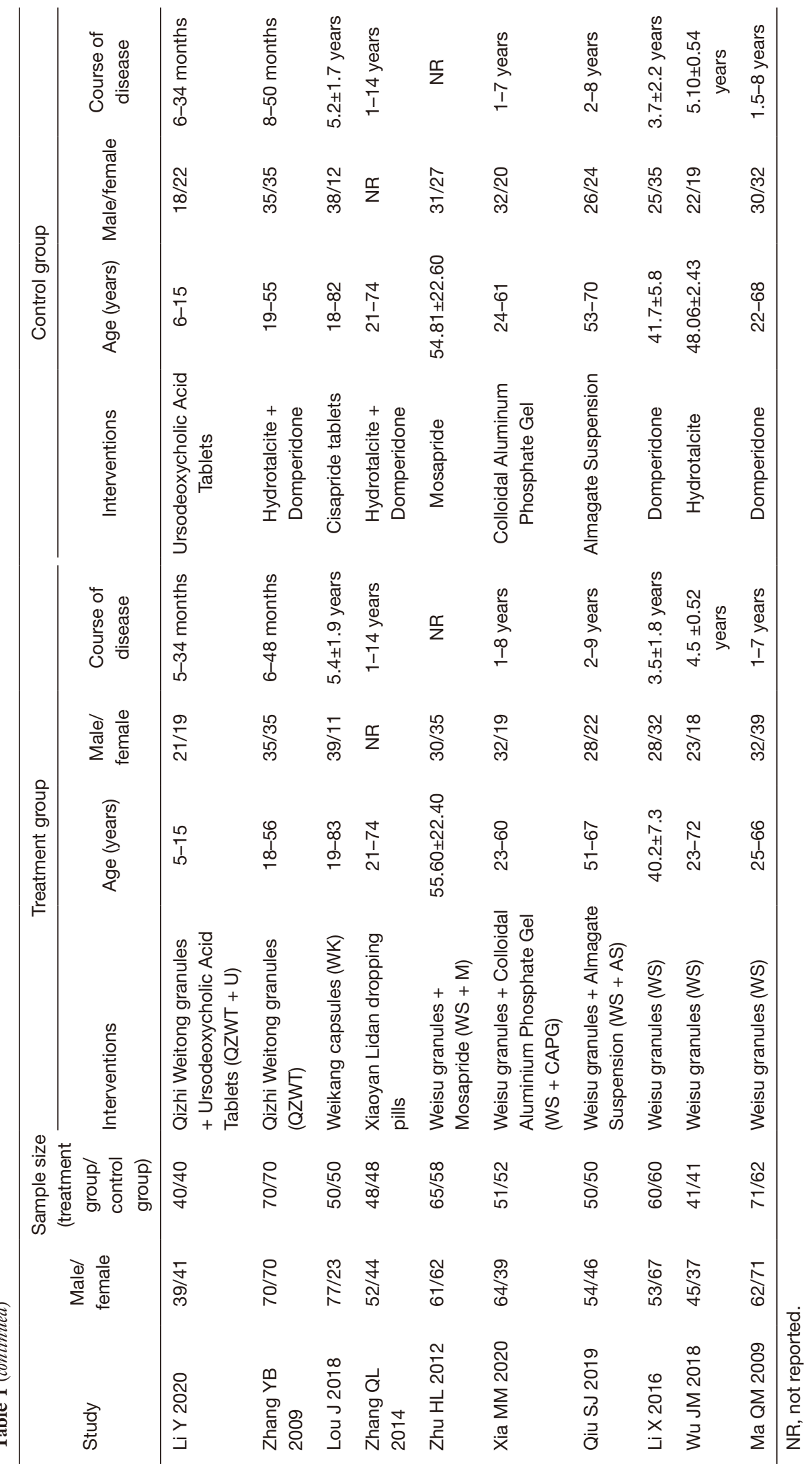




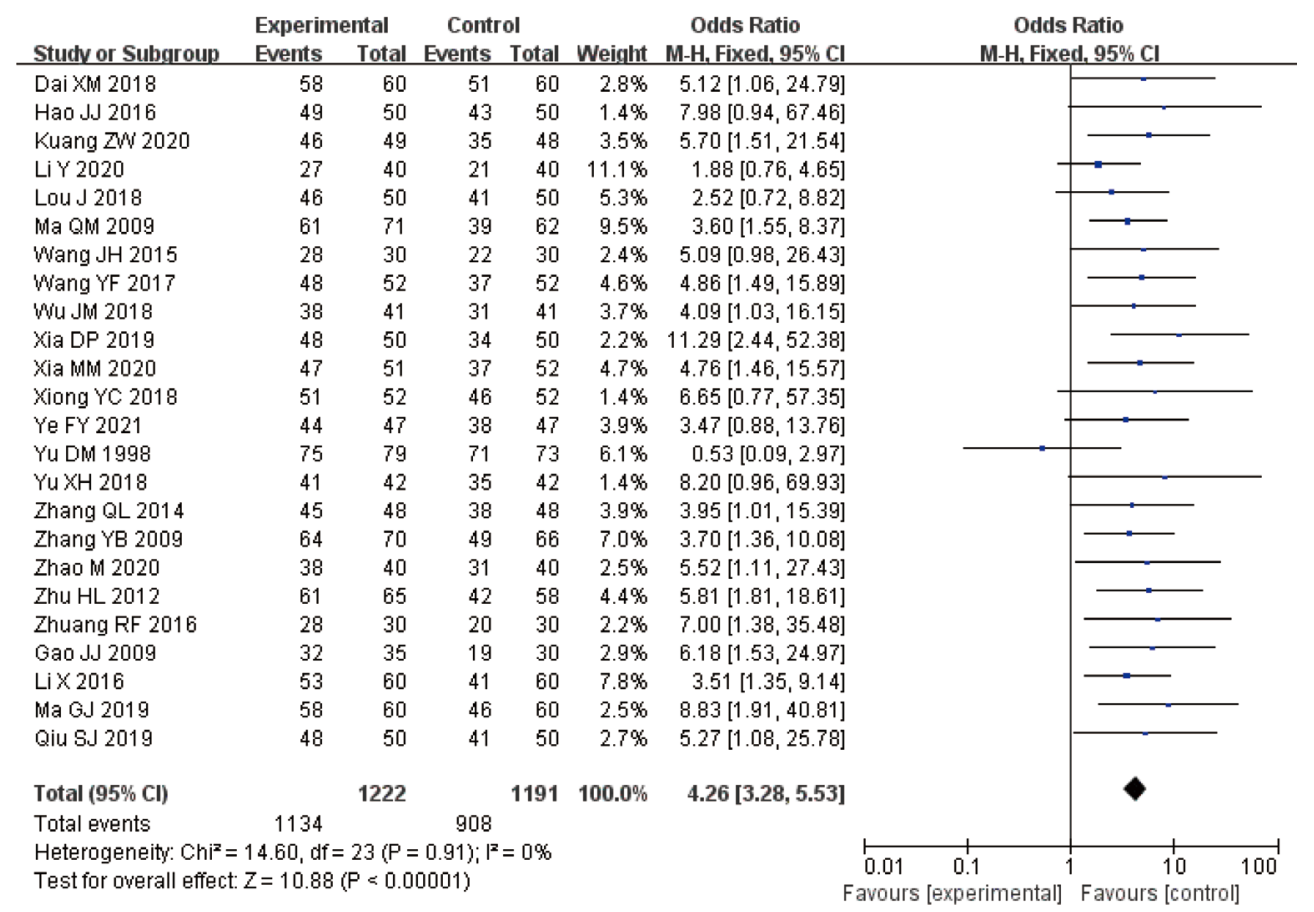

Figure 4 Forest plots of efficiency.

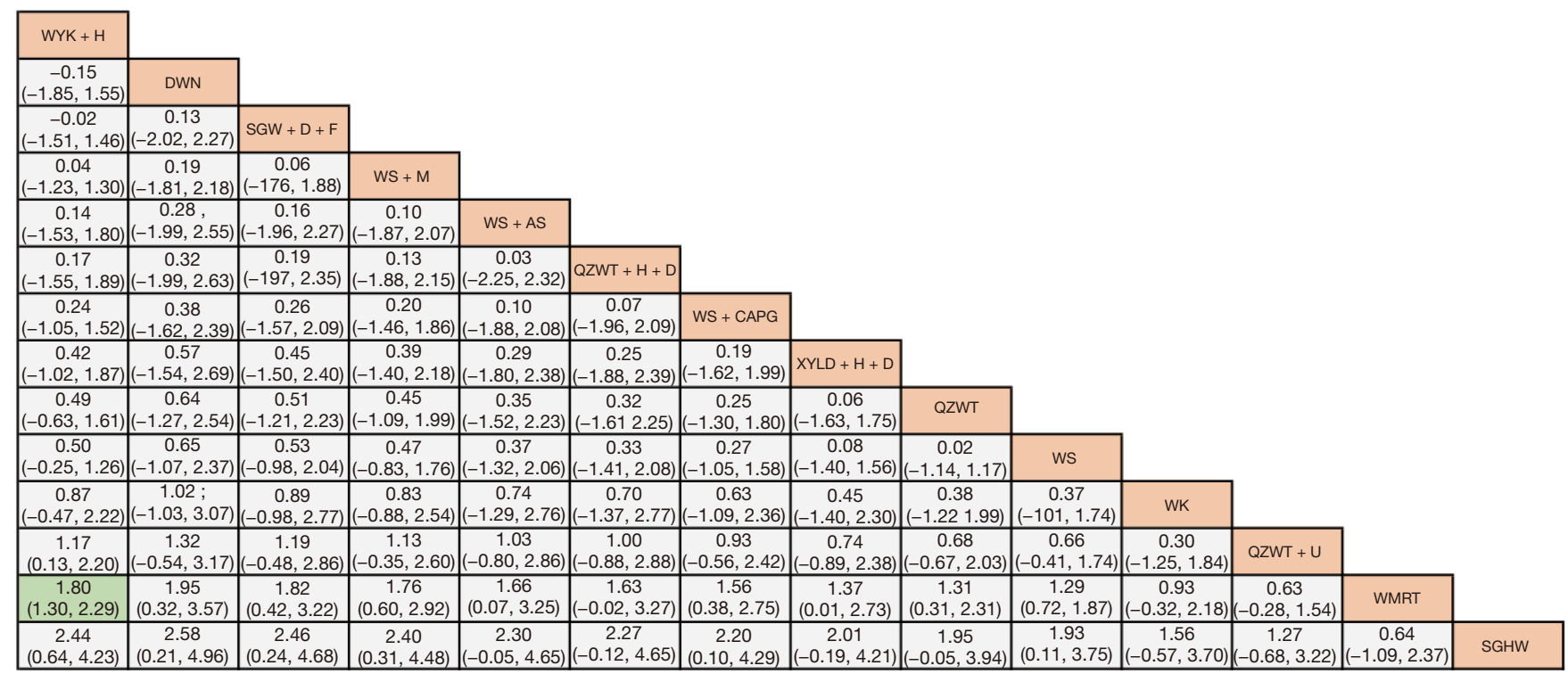

Figure 5 League table of efficient. 

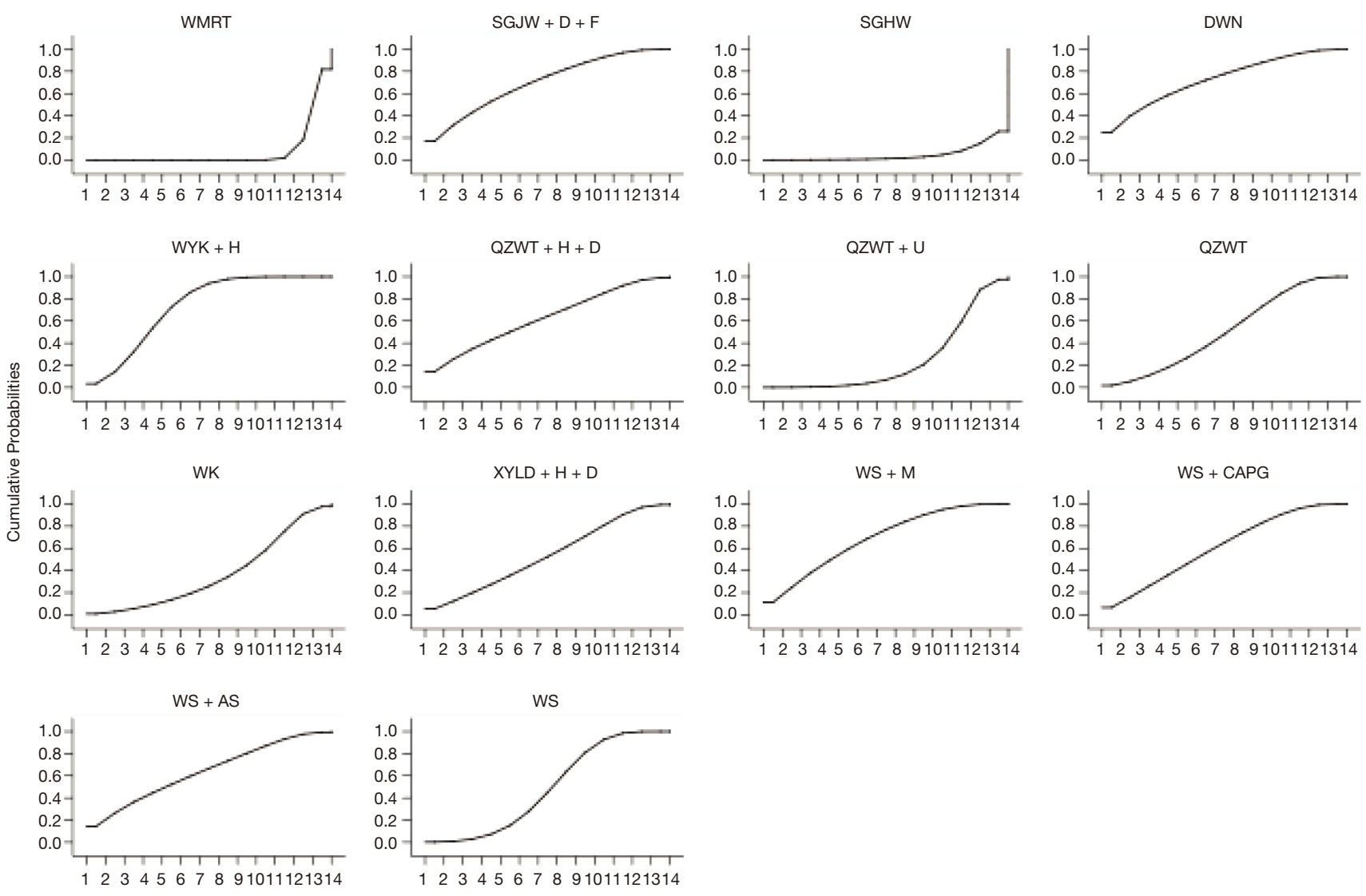

Graphs by Treatment

Figure 6 Cumulative probability of total effective rate.

Mosapride) (SUCRA =68.9), WS + AS (Weisu granules + Almagate Suspension) (SUCRA $=63.9)$, QZWT + H + D (Qizhi Weitong granules + Hydrotalcite + Domperidone) (SUCRA =62.4), WS + CAPG (Weisu granules + Colloidal Aluminium Phosphate Gel) (SUCRA =61.1), XYLD $+\mathrm{H}+\mathrm{D}$ (Xiaoyan Lidan dropping pills + Hydrotalcite + Domperidone) (SUCRA =53.7), QZWT (Qizhi Weitong granules) (SUCRA $=50.5$ ), WS (Weisu granules) (SUCRA =49.0), WK(Weikang capsules) (SUCRA =36.7), QZWT + U (Qizhi Weitong Granule + Ursodeoxycholic Acid Tablets) (SUCRA =25.2), WMRT (Routine treatment of western medicine) (SUCRA =7.9), and SGHW (Shugan Hewei pills) (SUCRA =4.7) (see Figure 6). Stata software was used to draw a comparison-correction chart for the included studies to evaluate the small sample effect. As Figure 7 shows, the research was roughly symmetrically distributed on both sides of the midline, indicating that a small sample effect was less likely to exist.

\section{Secondary outcomes}

There were 3 secondary outcome indicators in our study; that is, the recurrence rate, the TCM symptom score, and the gastroscopic mucosal severity score. Five of these interventions reported recurrence rates. A network plot is shown in Figure 8. In the 10 pairwise comparisons produced by the network meta-analysis under the consistency model, the differences were not statistically significant (see Figure 9). According to the SUCRA curve chart, the 5 intervention measures were ranked in probability. The probability of obtaining the lowest recurrence rate was ranked as follows: SGHW (SUCRA =73.9), QZWT (SUCRA =56.3), WYK + H (SUCRA $=52.0)$, SGJW + D + F (SUCRA =44.7), and WMRT (SUCRA =23.1) (see Figure 10). Due to the relatively small number of studies, no publication bias test was performed.

Various studies have compared TCM symptom scores. Due to the small number of interventions involved in the 


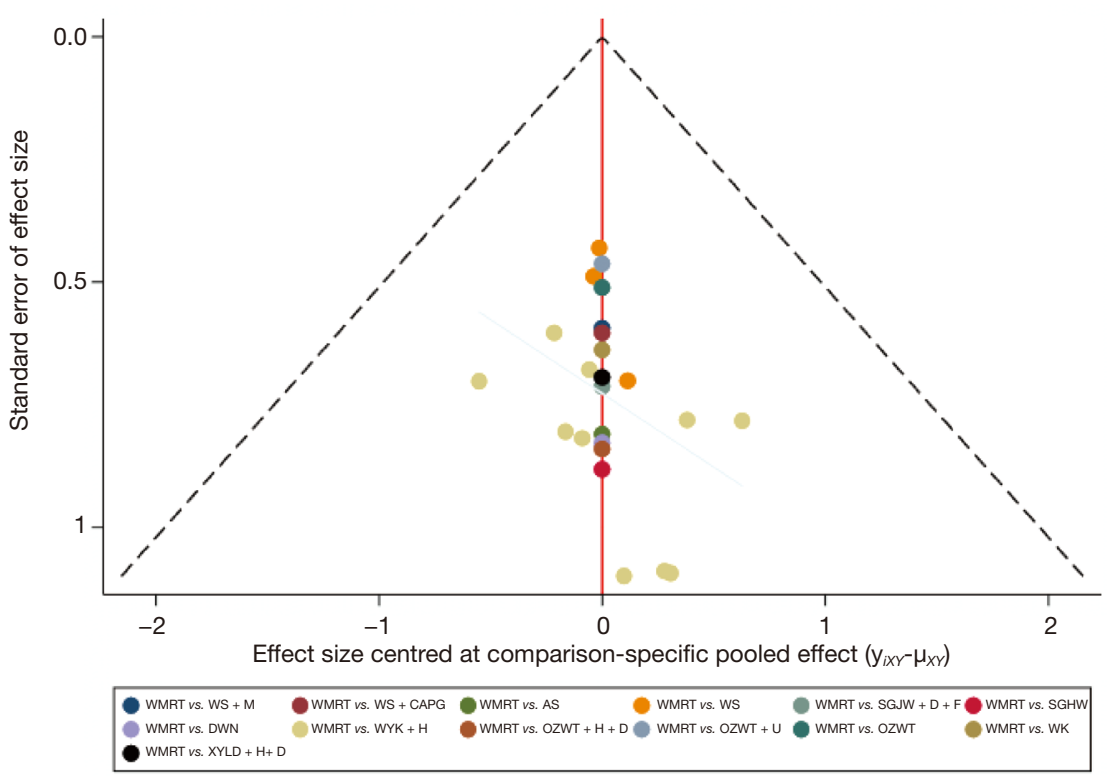

Figure 7 Funnel chart comparing the effectiveness of 14 interventions in the treatment of BRG.

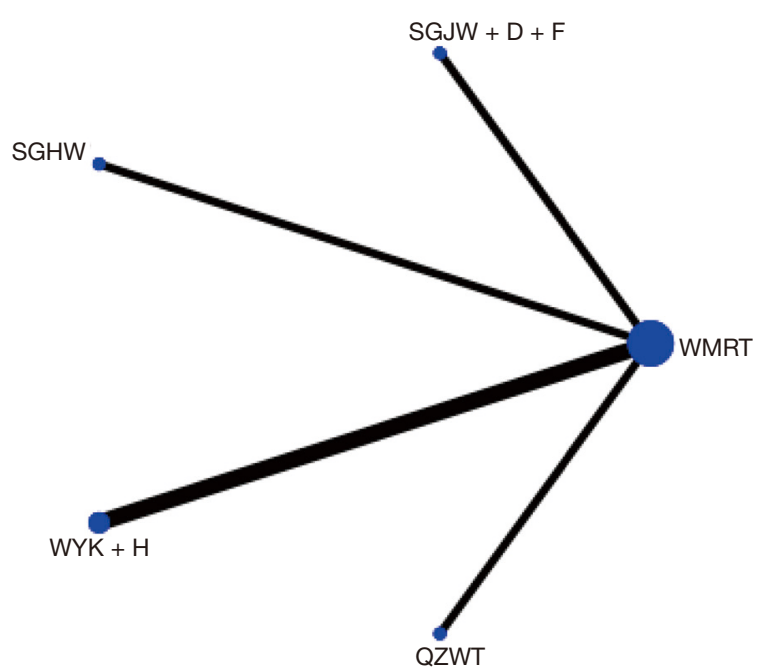

Figure 8 Network of eligible comparisons of the efficacy of recurrence rate.

present study, only a direct comparison meta-analysis could be performed. Three studies showed that the efficacy of the TCPMs in relieving the symptoms of stomach pain was better than that of conventional Western medicines $[\mathrm{MD}=-0.92,95 \% \mathrm{CI}(-1.49,-0.35)]$ (see Figure 11). Four studies showed that TCPMs were better than conventional Western medicines [MD $=-0.31,95 \%$ CI $(-0.44,-0.19)$ ] (see Figure 12). Four studies report that the efficacy of the
TCPMs was better at relieving the symptoms of abdominal distension than that of conventional Western medicines $[\mathrm{MD}=-0.58,95 \% \mathrm{CI}(-0.98,-0.18)]$ (see Figure 13). Three studies reported that the efficacy of TCPMs was better than that of conventional Western medicines at relieving acid reflux symptoms $[\mathrm{MD}=-0.30,95 \% \mathrm{CI}(-0.41,-0.19)]$ (see Figure 14). Five studies reported that the efficacy of TCPMs was better than that of conventional Western medicines at relieving belching symptoms $[\mathrm{MD}=-0.57,95 \% \mathrm{CI}(-0.72$, -0.41)] (see Figure 15).

Five studies comparing the scores of mucosal hyperemia on gastroscopy after treatment, revealed that after treatment, the score of the experimental group was significantly different to that of the control group [MD $=-0.56,95 \%$ CI $(-0.71,-0.40)]$ (see Figure 16). A comparison of 4 studies showed that the score of gastroscopic edema in the experimental group was significantly improved compared to that of the control group after treatment $[\mathrm{MD}=-0.82$, 95\% CI (-1.07, -0.57)] (see Figure 17). A comparison of 5 studies showed that the gastroscopic erosion score of the experimental group showed a significant improvement compared to that of the control group [MD $=-0.66,95 \% \mathrm{CI}$ $(-0.86,-0.46)]$ (see Figure 18).

\section{Discussion}

BRG is a disease in which a variety of factors cause bile 


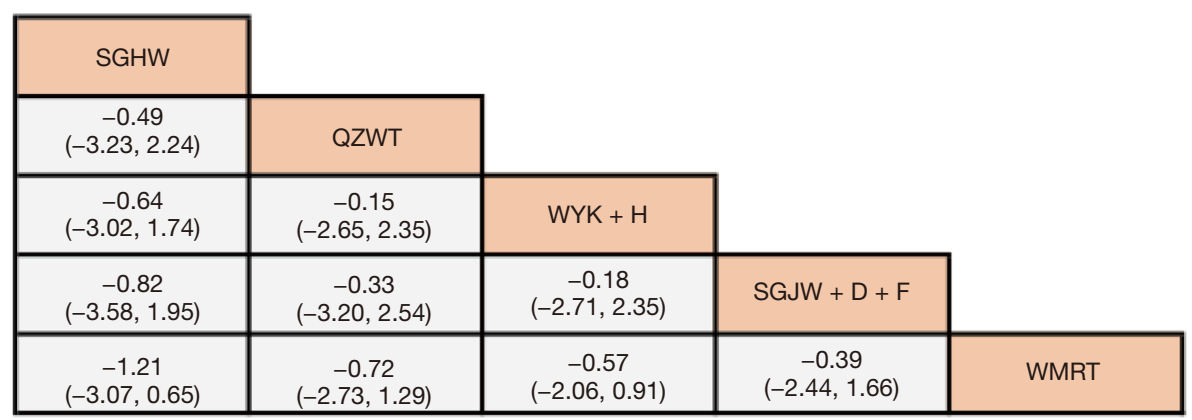

Figure 9 League table of recurrence rate

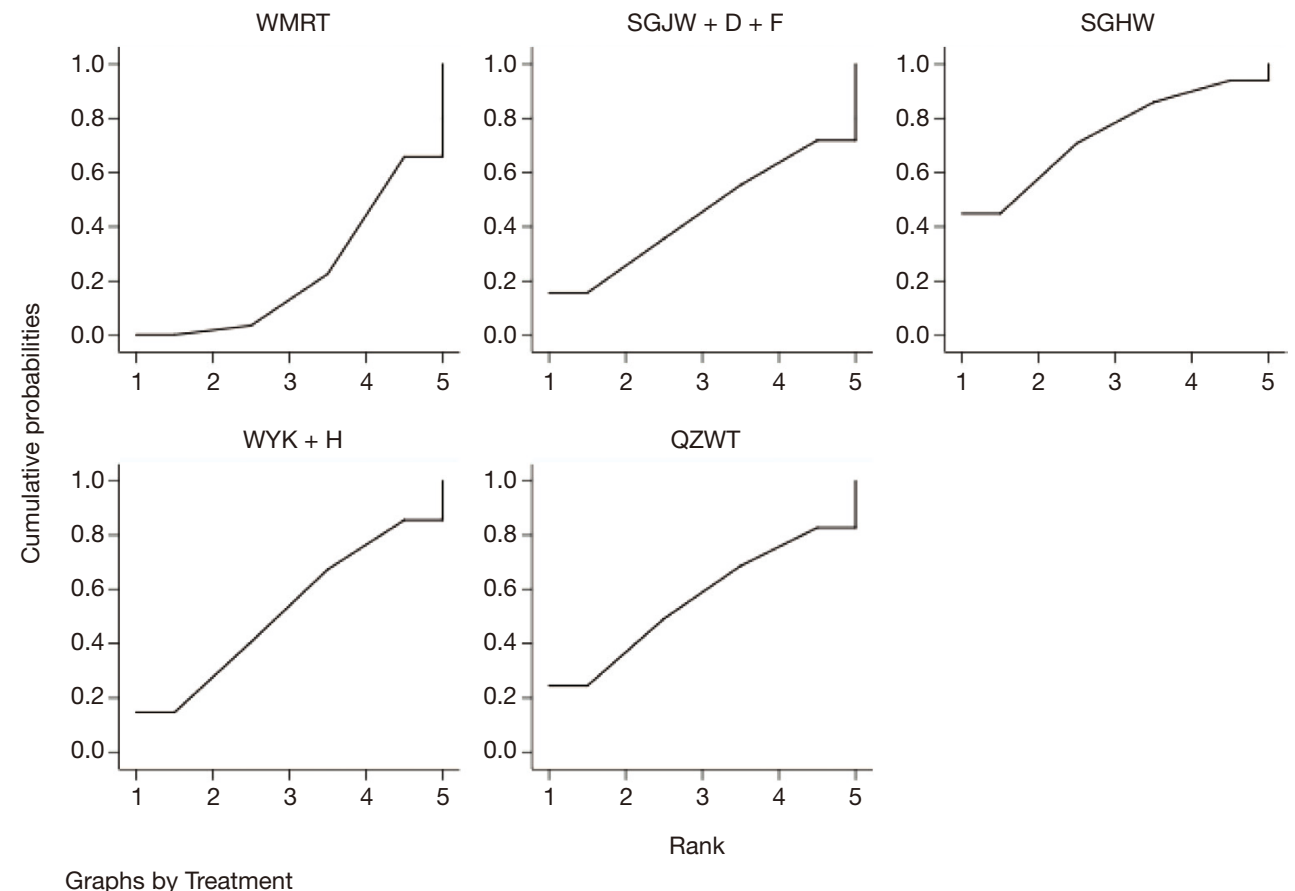

Figure 10 Cumulative probability of the recurrence rate.

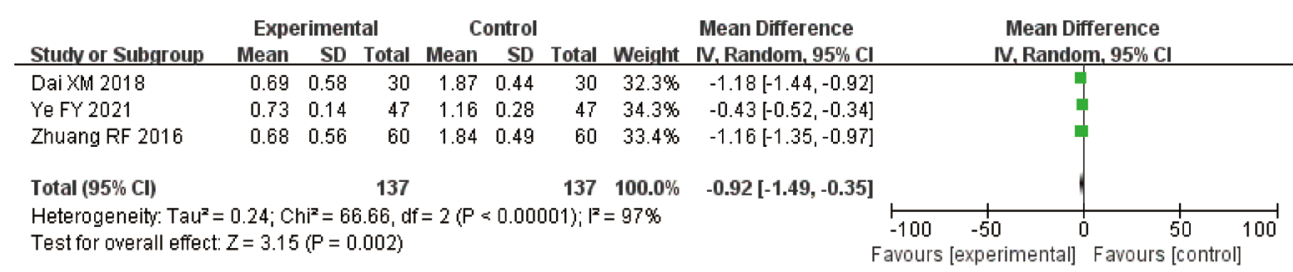

Figure 11 Forest plot of stomach pain.

to flow back into the stomach, thereby weakening or destroying the gastric mucosal barrier function, causing the gastric mucosa to be affected by digestive juice and bile acid, resulting in edema, congestion, erosion, and other diseases (36). Under repeated bile acid exposure, the gastric mucosa may also show atrophy, intestinal metaplasia, 


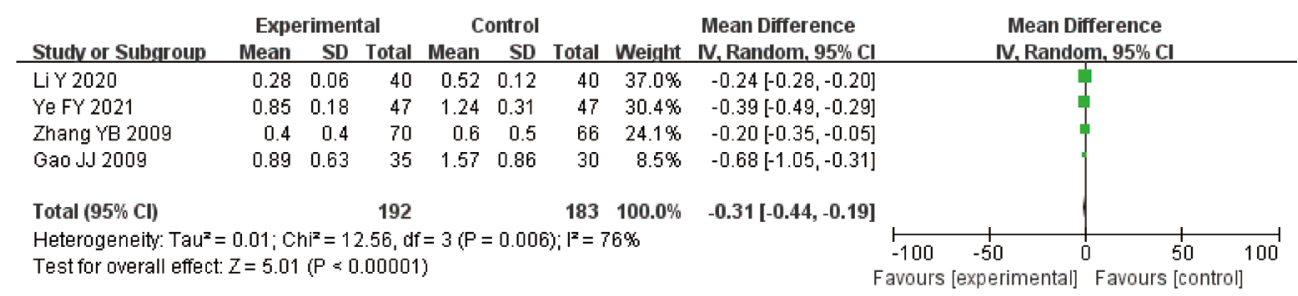

Figure 12 Forest plot of bloating.

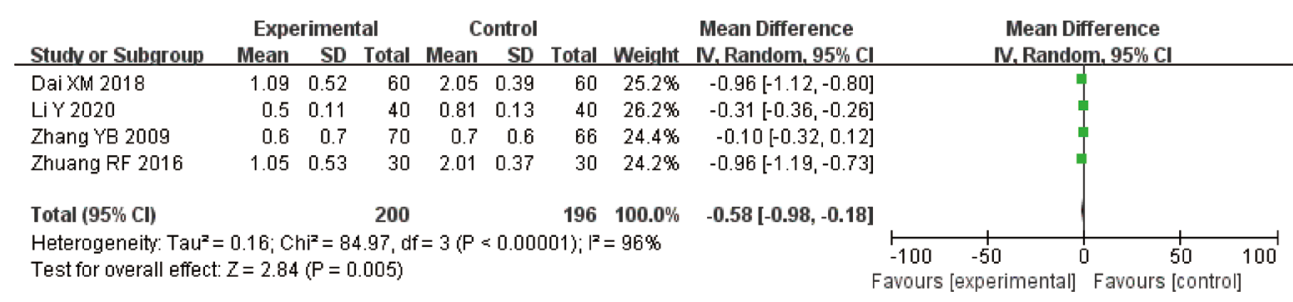

Figure 13 Forest plot of abdominal distention.

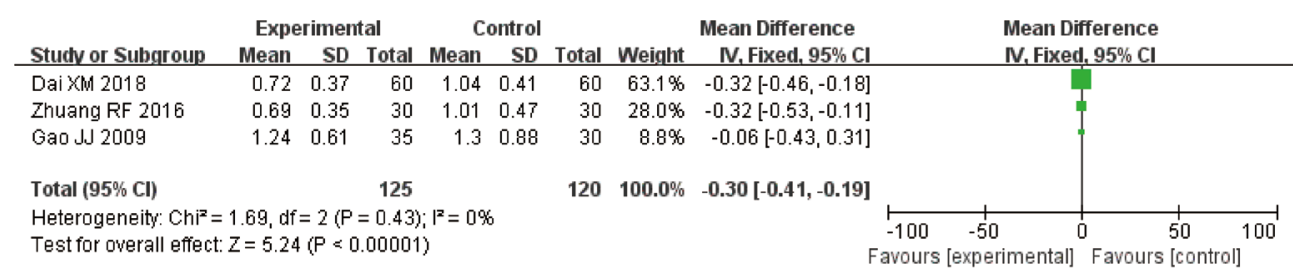

Figure 14 Forest plot of acid reflux.

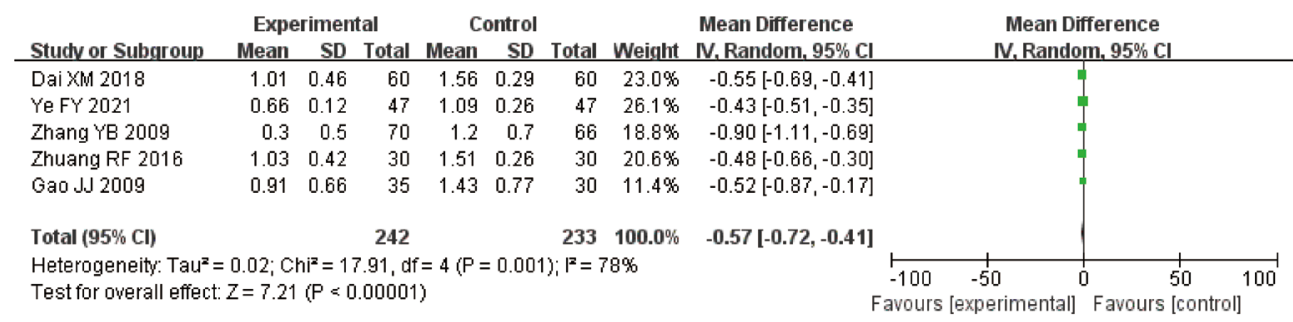

Figure 15 Forest plot of belching.

dysplasia, and cancer. Studies have also shown that there is a clear correlation between the severity of bile reflux and the severity of distal esophageal inflammation. Patients with more bile reflux may experience more severe esophageal mucosal damage (37). Currently, there are no drugs in clinical practice that can be specifically used to target bile reduction. A commonly used Western medicine is gastric mucosal protective agent-hydrotalcite, which promotes the inactivation of most of the pepsin while neutralizing gastric acid, thereby promoting the healing of the ulcer surface of the gastric mucosa and thus protecting the gastric mucosa (38). Some patients use combination therapy, including gastrointestinal motility drugs, domperidone and mosapride, to promote gastrointestinal peristalsis, reduce reflux, and reduce the time that refluxed bile and pancreatic juice stay in the stomach (39). However, taking these drugs 


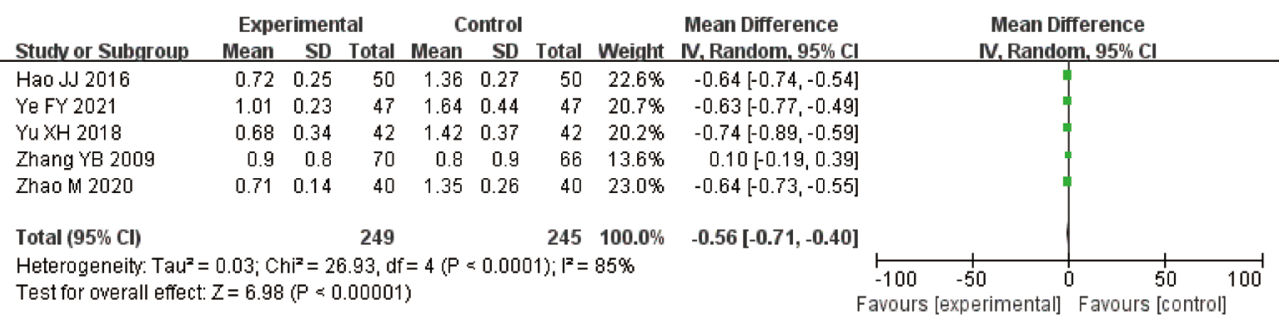

Figure 16 Forest plot of congestion.

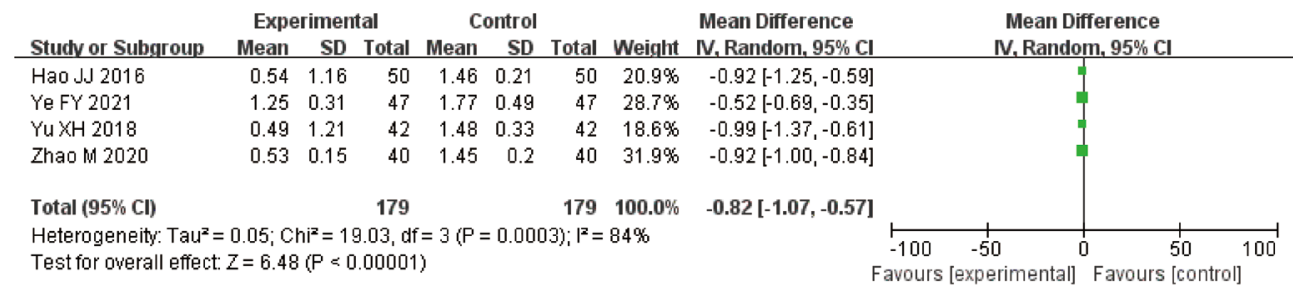

Figure 17 Forest plot of edema.

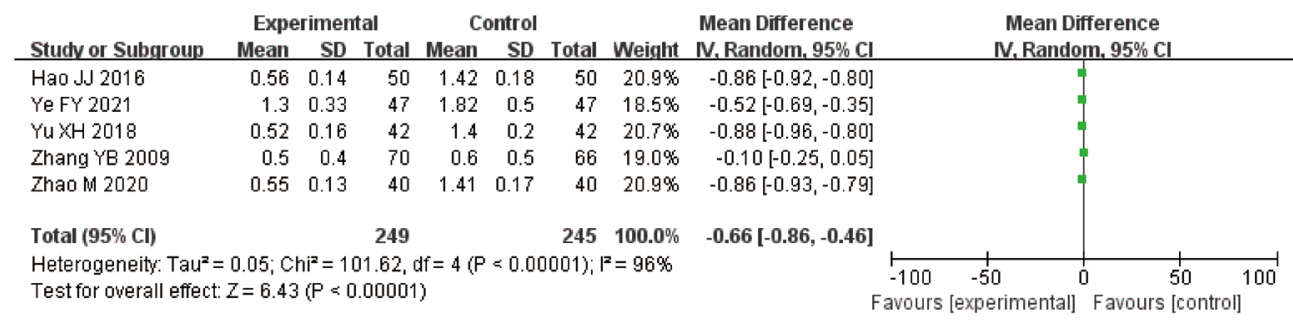

Figure 18 Forest plot of erosion.

for a long time may increase the incidence of adverse reactions in patients, such as diarrhea and vomiting, and relapse can easily occur.

Previous studies have shown that Chinese patent medicines have excellent clinical effects in the treatment of BRG, but there is still a lack of direct and indirect comparisons between the effects of various Chinese patent medicines. This study undertook a network meta-analysis to systematically evaluate the curative effect of 8 types of TCPMs. The results showed that Weiyankang capsules combined with hydrotalcite had the best curative effect in the treatment of bile reflux among the 14 interventions. Weiyankang capsules generally comprise the following 6 traditional Chinese medicines: white peony root, galangal, licorice, cassia twig, Bupleurum, and Coptis. The addition or removal of the Guizhi decoction has the effect of relieving pain and soothing the liver and stomach. Modern pharmacological studies have shown that it has good analgesic, anti-inflammatory, and anti-infective effects, greatly improves gastric mucosal lesions, and promotes gastric mucosal repair and regeneration (25). The Shugan Hewei pill is a proprietary Chinese medicine. It had the lowest recurrence rate among the 5 studies that reported recurrence rates. The ingredients of Shugan Hewei pills are Amomum villosum, Magnolia officinalis, Angelica, Citrus aurantium, green peel, tangerine peel, turmeric, and white peony. Among them, Amomum villosum, Magnolia officinalis, Citrus aurantium, Qingpi, and tangerine peel are mainly responsible for regulating qi and reducing adversity. Turmeric and Radix Paeoniae Rubra can soothe the liver, relieve depression, and relieve pain. Angelica nourishes yin and promotes blood circulation. The composition of the prescription has the effect of soothing the liver, regulating qi, and neutralizing vital energy. 
In contrast to Western medicine, TCPM is holistic in the manner of traditional Chinese medicine, which not only effectively cures the clinical symptoms of patients but is less toxic and has fewer side effects. Compared with traditional Chinese medicine decoctions, TCPMs are more convenient to take, and patients are more compliant in taking their medication. At present, there are many kinds of TCPMs for the treatment of BRG. However, the constituents of TCPM are fixed, and it is impossible to customize the medication for individual patients by adding or removing components. It is expected that more targeted TCPMs will be developed in the future that will permit customized treatment based on an improved classification system of symptoms and diseases.

\section{Limitations}

This study had a number of limitations. First, none of the studies included in the meta-analysis explained blinding and allocation concealment. Second, the included studies had a relatively short observation period (most of the studies focused on 1 to 2 months of treatment); thus, there was a lack of long-term efficacy evaluations. Third, there are very few reports on the adverse effects of the interventions in the included studies, thus there was a lack of evaluations or comparisons of the safety of the different drugs in the treatment of BRG.

\section{Conclusions}

This network meta-analysis showed that compared to conventional Western medicines, TCPMs can significantly improve the curative effect of BRG. Based on the present study, among the various TCPM choices available, the Weiyankang capsule provided the best treatment strategy. To date, very few studies have reported on adverse reactions and recurrence rates; thus, no comparisons were able to be made. However, it is clear that TCPMs have certain advantages in improving symptoms. In addition, the quality of this study was low, and there was a certain risk of bias. In the future, more high-quality RCTs need to be conducted to improve the reliability of the conclusions drawn in this paper.

\section{Acknowledgments}

Funding: The 3rd Traditional Chinese Medicine Master Inheritance Studio and the National Famous Traditional Chinese Medicine Inheritance Studio Construction Project [No. Liaozhongyiyaohanzi(2018)76)]. Specialized project to improve the evidence-based ability of specialty diseases in the field of traditional Chinese medicine digestion (chronic atrophic gastritis) [No. 2019XZZX-XH008]. Study on the Living Inheritance of Shengjing Spleen and Stomach Academic School [No. LiaoWeiZongHeZi(2021)19].

\section{Footnote}

Reporting Checklist: The authors have completed the PRISMA reporting checklist. Available at https://dx.doi. org/10.21037/apm-21-1307

Conflicts of Interest: All authors have completed the ICMJE uniform disclosure form (available at https://dx.doi. org/10.21037/apm-21-1307). The authors have no conflicts of interest to declare.

Etbical Statement: The authors are accountable for all aspects of the work in ensuring that questions related to the accuracy or integrity of any part of the work are appropriately investigated and resolved.

Open Access Statement: This is an Open Access article distributed in accordance with the Creative Commons Attribution-NonCommercial-NoDerivs 4.0 International License (CC BY-NC-ND 4.0), which permits the noncommercial replication and distribution of the article with the strict proviso that no changes or edits are made and the original work is properly cited (including links to both the formal publication through the relevant DOI and the license). See: https://creativecommons.org/licenses/by-nc-nd/4.0/.

\section{References}

1. Chan DC, Fan YM, Lin CK, et al. Roux-en-Y reconstruction after distal gastrectomy to reduce enterogastric reflux and Helicobacter pylori infection. J Gastrointest Surg 2007;11:1732-40.

2. Kawiorski W, Herman RM, Legutko J. Current diagnosis of gastroduodenal reflux and biliary gastritis. Przegl Lek 2001;58:90-4.

3. Li T, Pu F, Wang K, et al. Clinical thoughts on treatment of reflux esophagitis from shaoyin heat transformation. Longhua Chin Med 2020;3:23.

4. Chen LY, Wang LH, Wang HM, et al. A survey of prevalence of bile reflux gastritis. Chinese Journal of Integrated Traditional and Western Medicine on Digestion 2015;23:108-11. 
5. Tel'nykh YV, Abgadzhava EZ, Kon'kov MY. Biliary refluxgastritis: etiology, pathogenesis and modern principles of treatment. Klin Med (Mosk) 2016;94:454-7.

6. Zhou YB, Chen DF. Current status of bile reflux gastritis. Chin J Gastroenter Hepatol 2019;28:689-92.

7. Cheng SH. The effect of hydrotalcite combined with Weiyankang capsule in the treatment of bile reflux gastritis. China Health Nutrition 2020;30:229.

8. Zhang HM, Zhang XQ. Research Progress of Chinese Medicine In the Treatment of Bile Reflux Gastritis.AsiaPacific Traditional Medicine 2021;17:198-201.

9. Lou J, Zhao YH. Clinical Observation of Weikang Capsule in Treating Bile Reflux Gastritis. China's Naturopathy 2018;26:28-9.

10. Xie GZ, Xu DS. Danweishu Granule in the Treatment with Bile Reflux Gastritis. Acta Chinese Medicine 2012;27:1184-1185.

11. Zhuang RF, Gu QH. Clinical effect of bile reflux gastritis with gall bladder and stomach deficiency type treated with Dan-Wei-Ning granules. Lishizhen Medicine and Materia Medica Research 2016;27:2940-3.

12. Melsen WG, Bootsma MC, Rovers MM, et al. The effects of clinical and statistical heterogeneity on the predictive values of results from meta-analyses. Clin Microbiol Infect 2014;20:123-9.

13. Liberati A, Altman DG, Tetzlaff J, et al. The PRISMA statement for reporting systematic reviews and meta-analyses of studies that evaluate health care interventions: explanation and elaboration. J Clin Epidemiol 2009;62:e1-34.

14. Gao JJ. The clinical observation of Shuganjianwei pill and western medicine in treating bile reflux gastritis. Journal of Clinical Rational Drug Use 2009;2:24-5.

15. Yu DM, Chen XH. Shuganhewei Pills in Treating 152 Cases of Bile Reflux Gastritis. Journal of Shanghai Tiedao University 1998;9:64-5.

16. Ma GJ. Analysis of the clinical efficacy of hydrotalcite combined with Weiyankang capsule in the treatment of bile reflux gastritis. Diet 2019;12:61.

17. Xia DP, Shen AQ. The clinical effect of hydrotalcite combined with Weiyankang capsule in the treatment of bile reflux gastritis. China Practical Medicine 2019;14:97-8.

18. Xiong YC. Clinical observation of hydrotalcite combined with Weiyankang capsule in the treatment of bile reflux gastritis. Journal of North Pharmacy 2018;15:25-6.

19. Ye FY. The clinical efficacy and safety of hydrotalcite combined with Weiyankang capsule in the treatment of bile reflux gastritis. Journal of clinical rational drug use 2021;14:115-7.

20. Yu XH. Efficacy analysis of hydrotalcite combined with Weiyankang capsule in the treatment of bile reflux gastritis. Psychologies 2018;9:261.

21. Wang YF. Clinical Efficacy of Hydrotalcite Combined with Weiyankang Capsule in Treating Bile Reflux Gastritis. China Pharmaceuticals 2017;26:32-4.

22. Dai XM. Effect and safety of Weiyankang Capsule in adjuvant treatment of bile reflux gastritis. China Modern Medicine 2018;25:88-91.

23. Hao JJ, Miao HJ. Clinical study on Weiyankang Capsules combined with hydrotalcite in treatment of bile reflux gastritis. Drugs \& Clinic 2016;31:1542-6.

24. Zhao M. Effects of Weiyankang capsules combined with Aluminum magnesium carbonate on patients with bile reflux gastritis. Medical Journal of Chinese People's Health 2020;32:79-81.

25. Kuang ZW. Clinical study of Weiyankang capsule combined with hydrotalcite in the treatment of patients with bile reflux gastritis. Strait Pharmaceutical Journal 2020;32:123-4.

26. Wang JH, Zhang L. Observation on the Treatment of 30 Cases of Bile Reflux Gastritis with Integrated Traditional Chinese and Western Medicine. Journal of Practical Traditional Chinese Medicine 2015;31:746.

27. Li Y, Zhang F, Zhou JM. Effect of Qizhiweitong Granules Combined with Ursodeoxycholic Acid in the Treatment of Bile Reflux Gastritis with Disharmony of the Liver and Stomach. Shanxi Journal of Traditional Chinese Medicine 2020;41:50-2.

28. Zhang Y B, Deng EL, Peng YF, et al. Observation of bile reflux gastritis with incoordination between the liver and stomach treated by Qizhiweitong Granule. Hebei Journal of Traditional Chinese Medicine 2009;31:1301-3.

29. Zhang QL. Analysis of the clinical efficacy of Xiaoyanlidan dripping pills combined with domperidone and hydrotalcite in the treatment of bile reflux gastritis. The Journal of Medical Theory and Practice 2014;27:611-2.

30. Zhu HL. Treatment of 62 Cases of Bile Reflux Gastritis with Integrated Traditional Chinese and Western Medicine. Chinese Community Doctors 2012;14:183.

31. Xia MM, Li CY, Liu JH. Clinical study on Weisu Granules combined with Colloidal Aluminum Phosphate Gel in treatment of bile reflux gastritis. Drugs \& Clinic 2020;35:2043-6.

32. Qiu SJ, Yu T. Clinical Study of Weisu Granules Combined with Aluminum Magnesium Suspension on Reflux 
Gastritis. Shanxi Medical Journal 2019;48:2035-7.

33. Li X. Weisu Granule treating 120 cases bile reflux gastritis. World Latest Medicine Information 2016;16:170-1.

34. Wu JM, Xie HJ, Luo G ,et al. Clinical efficacy of Weisu granule in treating bile reflux gastritis and its influence on CRP. Psychological Doctor 2018;24:81-2.

35. Ma QM. Observation on Curative Effect of Weisu Granule on Bile Reflux Gastritis . Shanghai Journal of Traditional Chinese Medicine 2009;43:47, 50.

36. Sifrim D. Management of bile reflux. Gastroenterol Hepatol (N Y) 2013;9:179-80.

37. Jiang ZY. Study on the clinical effect of hydrotalcite in

Cite this article as: He M, Wang Q, Liu L, Bai G. Traditional Chinese patent medicine for bile reflux gastritis: a systematic review and network meta-analysis. Ann Palliat Med 2021;10(7):7721-7735. doi: 10.21037/apm-21-1307 the treatment of bile reflux gastritis. Journal of Clinical Medical Literature 2018;5:101, 103.

38. LI ZT. Clinical effect of hydromagnesium carbonate chewable tablets combined with mosapride in the treatment of bile reflux gastritis. Henan Medical Research 2020;29:3761-2.

39. CAI Q. Analysis of the clinical effect of domperidone combined with hydromagnesium carbonate in patients with bile reflux gastritis. China Practical Medicine 2021;16:127-8.

(English Language Editor: L. Huleatt) 